\title{
Diálogo con Europa, diálogo con el pasado. Antepasados y tradición entre la nobleza colonial nahua
}

\author{
Julia MADAJCZAK y Justyna OlKo \\ Facultad de «Artes Liberales», Universidad de Varsovia \\ julia@al.uw.edu.pl jolko@al.uw.edu.pl
}

Recibido: 15 de julio de 2014

Aceptado: 8 de agosto de 2014

\begin{abstract}
RESUMEN
En este trabajo presentamos un acercamiento a los modos de referirse al pasado en los testimonios dejados por los miembros de la nobleza colonial nahua. La lectura de las fuentes nos permite distinguir dos perspectivas -intelectual y pragmática- asociadas respectivamente con el proyecto de unir las tradiciones históricas indígena y europea y con la búsqueda de argumentos en la lucha por tierras y privilegios. El enfoque del artículo son los conceptos nahuas de «tradición» y «antepasados» (huehuetqueh), manifiestos en ambas perspectivas. $\mathrm{Al}$ analizar el vocabulario, retórica e iconografía intentamos entender el significado de estos conceptos dentro de la cultura nahua prehispánica y su modificación bajo las circunstancias coloniales, tanto como su papel en la continuación de la identidad nahua.
\end{abstract}

Palabras clave: Cultura nahua, nobleza, tradición, cambio y continuidad.

\section{A Dialogue with Europe, a Dialogue with the Past. Ancestors and Tradition among the Colonial Nahua Nobility}

\begin{abstract}
In this paper we discuss the ways in which the Nahua nobility referred to the past as reflected in the testimonies of the colonial period. The reading of sources allows us to distinguish two perspectives: intellectual and pragmatic. While the first of them was closely linked to attempts of combining the indigenous and European historical traditions, the second one involved the quest for arguments in an ongoing struggle for land and privileges. We approach this complex topic focusing on the Nahua concepts of «tradition» and «ancestors» (huehuetqueh), common for both perspectives. Our analysis of the terminology, rhetoric and iconography refines the understanding of the functions and meaning of these concepts in precontact Nahua culture, their modifications under colonial circumstances, as well as the role they played in the continuity of Nahua identity.
\end{abstract}

Key words: Nahua culture, nobility, tradition, change and continuity.

Sumario: 1. Planteamiento del problema. 2. La memoria y el pasado a través de la terminología náhuatl. 3. Hacia una perspectiva intelectual: reevaluación de la tradición indígena y diálogo con Europa. 4. Diálogo con Europa. 5. Una perspectiva pragmática. 6. Conclusiones. 7. Referencias documentales. 8. Referencias bibliográficas.

\section{Planteamiento del problema}

La continuidad de la nobleza indígena en la época colonial implicaba una tensión permanente entre los esfuerzos por conservar la base de poder prehispánica y los reajustes complejos a la nueva realidad. Fue una lucha por, como lo planteó José Luis de Rojas (2010), «cambiar para que yo no cambie», para que se mantuviera el lugar privilegiado de la elite indígena. Esta tensión implicaba varias formas de diálogo asociado a un espectro muy amplio de posibles estrategias tanto ideológicas como prácticas entre las tradiciones del pasado y un enorme bagaje de origen europeo. Este último no 
solo ofrecía numerosas oportunidades e imponía soluciones abiertas a negociación, aunque encubierta, sino que también planteaba numerosos retos culturales, ideológicos, económicos y políticos. Dentro de una gran heterogeneidad de «diálogos» entre los dos mundos, se pueden identificar dos perspectivas más generales: una intelectual y otra pragmática, que, como vamos a demostrar, no solo se complementan, sino que además manifiestan puntos de encuentro y convergencia. Fundamental para las dos es la importancia y papel de los antepasados.

La primera perspectiva implica el reto de encajar la tradición indígena y los mitos de etnogénesis en la historia universal del mundo y los conceptos europeos. Los esfuerzos por encontrar los puntos comunes entre las dos tradiciones se reflejan en las narraciones históricas basadas en la cuenta de tiempo cristiana y nativa de manera paralela, identificación de analogías entre la tradición bíblica e indígena y reevaluación positiva del pasado basada, por ejemplo, en la afinidad percibida entre el paganismo de la antigüedad europea y el paganismo prehispánico. Los paralelos entre los habitantes de América y los paganos griegos y romanos, ofrecían la posibilidad de una «etnología comparativa» donde se podían transcender las distancias temporales y las diferencias culturales (Seth 2010: 55). Por otro lado, la visión pragmática se apoyó en la identificación tradicional con el altepetl, o un estado indígena, y también con una identidad a nivel de familia o linaje. Aquí el papel de los antepasados adquirió no sólo valor conceptual, sino también práctico: ¿en qué medida pudieron haber sido útiles en el contexto colonial, en la legitimación de poder y estado de posesión o para lograr unos fines políticos y económicos nuevos?

Una mirada cuidadosa a ambas perspectivas con un enfoque en el papel de los antepasados, nos permite también acercarnos a un concepto más sutil del pensamiento nahua: lo que podemos llamar «tradición». Nuestro punto de partida consiste en ver «tradición» como parte de un sistema cultural (tal como lo entiende C. Geertz 1999). En consecuencia, no buscamos un equivalente exacto nahua del concepto de «tradición» europeo ni a nivel lingüístico ni ideológico. Por el contrario, tratamos de abordarlo desde una perspectiva local, en términos propios a la cultura nahua: ¿Qué vocablos náhuatl fueron utilizados en el contexto de «tradición»? ¿Qué se consideraba la fuente de la tradición, a qué propósitos servía y cómo pasaba a las generaciones siguientes? La búsqueda de las respuestas implica leer entre líneas las fuentes escritas y ver más allá de la mera representación en las pictóricas. Como cada concepto indígena, en el contacto con los españoles «tradición» se sometió a cambios tanto intencionales (p.ej. el mencionado proyecto de crear una historia europea-indígena), como inconscientes, llamados por J. Lockhart (1999: 99) «double mistaken identity».

\section{La memoria y el pasado a través de la terminología en náhuatl}

Uno de los términos claves dentro de la conceptualización nahua de la «tradición» era la palabra «huehueh», que significaba «viejo» o «anciano» (Molina 1977 II: 157r). Un huehueh gozaba de una posición crucial en la sociedad, guardando y transmitiendo a otros mensajes que contenían la riqueza cultural y la identidad. A los viejos se les admiraba y respetaba por su sabiduría y por la experiencia que acumulaban a lo largo 
de sus vidas, pero también por sus cualidades espirituales. En la cosmovisión nahua, la esencia caliente de origen divino que podría «almacenarse» en cada ser humano crecía con el tiempo, aumentando las capacidades de comunicarse con el más allá. «Huehueh ilama» (lit. «anciano anciana»), la metáfora náhuatl para fuego y humo - con los cuales las ofrendas humanas se transmitían hacia los dioses (Ruíz de Alarcón 1984: 166)- subrayaba el vínculo de los viejos con la mediación. Lo hacía también su papel de traspasar la tradición oral a través de los discursos llamados «huehuehtlahtolli» (lit. "palabras antiguas»), porque palabras y aliento contenían esencias divinas y servían de ofrenda a los dioses. Sin control, la fuerza enorme que adquirían los viejos, podía convertir a un hombre en un monstruo peligroso, pero bien controlada era indispensable para gobernar (López Austin 1984 I: 327-328). Como indica López Austin, fray Alonso de Molina traduce el sustantivo poseído «-huehuehuehyohuan» (lit. «varios ancianos de uno») por «mis ministros de justicia» o «los oidores o alcaldes de corte», mientras que el verbo «huehuehtlalia» (lit. «instalar a una persona vieja») quiere decir «dar cargo y oficio honroso a otro» (Molina 1977 II: 157r). En los documentos cotidianos se describe con el término «huehuetqueh» a las autoridades de tlaxilacalli, una sub-entidad administrativa de altepetl (Lockhart 1992: 44). Lo que parece interesante es que estos «ancianos» de tlaxilacalli no necesariamente eran de una edad avanzada. Un documento tlatelolca del año 1558 cita los testimonios de Martín Tochtli y Miguel Díaz, ambos de 60 años de edad, que fueron huehuetqueh cuarenta años atrás, siendo, evidentemente, jóvenes de veinte y tantos años (véase AGN Tierras, vol. 17, parte $2^{\text {a }}$, exp. 4, ff. 11-14). Esto sugiere que en los contextos políticos el término «huehuetqueh» no se refería a la edad de los oficiales sino a su capacidad de gobernar. En la cultura nahua «ser viejo» significaba «ser poderoso», en el sentido de guardar una gran cantidad de esencia divina que permitía mediar entre los hombres y los dioses y, gracias a esto, gobernar a la gente.

Otro concepto connotado por la palabra «huehueh» es pertenencia a los tiempos inmemorables. Junto con los guardianes de la identidad y la tradición, el nombre de «huehuetqueh» lo adquirían los antepasados que establecieron esta tradición siendo la propia fuente de la identidad. Lo confirma directamente uno de los discursos recopilados por fray Bernardino de Sahagún:

«¿Cuántas son las palabras guardadas, las que dan vida, las que son dignas de ser guardadas, las que dejaron al partir nuestros antepasados, los ancianos, las ancianas, las personas canas, canas de la cabeza?»${ }^{1}$ (Sahagún 2012 VI: 124-125).

En otro discurso la tradición oral se describe como «la funda, el cofre [la cosa secreta] que dejaron al partir los ancianos, las ancianas, los que andan canos, los que andan canos de la cabeza, los que andan flacos de vejez, los que son nuestros

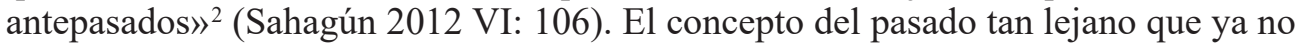
puede ser recordado con exactitud se desprende también de otros términos que incorporan la palabra «huehueh». Así, Huehuehteotl, el dios de fuego, gozaba del culto

\footnotetext{
1 «quexqujch in mopixtoc tlatolli, in nemoanj, in pialonj: in concauhteoaque in totechiuhcaoan in vevetque, in jlamatque, in tzonjztaque, in quaiztaque». Todas las traducciones del náhuatl son de las autoras.

2 «in toptli, in petlacalli: in concauhteoaque in vevetque, in jlamatque, in tzonjztaztivi, in quaiztaztivi, in pipinjxtivi in totechiuhcaoan».
} 
más antiguo en Mesoamérica, pero también estaba colocado en el centro del cosmos y en los principios del tiempo, siendo llamado «la madre y padre de los dioses» (Sahagún 2012 VI: 19). De la misma manera los «huehuehpipiltin» («nobles antiguos») procedían en línea recta del primer tlahtoani de los mexica, Acamapichtli, aunque ya se había olvidado su genealogía exacta:

«Aunque no se sabe quiénes eran los progenitores, los padres, cuáles eran los nombres de los que les engendraron [a los huehuepipiltin], se dice, sin embargo, que ellos de verdad eran los nietos del señor Acamapichtli, [su linaje] se termina con él. Por eso se les llama huehuehpipiltin» ${ }^{3}$ (Chimalpahin y Tezozomoc 1997: 116).

Resulta igual de llamativo que en los relatos históricos del siglo XVI de Chimalpahin (2003 y 2006), que conservan la forma de los anales tradicionales, el término huehueh sirva para distinguir tanto a los antepasados remotos, los huehuehchichimeca, como a los chichimecas que le eran contemporáneos, identificados con los habitantes de las zonas norteñas de la Nueva España.

Finalmente, el tercer aspecto importante de «lo antiguo» era la transmisión, ya tratada en el caso de los «huehuehtlahtolli», pero su importancia la encontramos destacada en la terminología económica. Como sugiere J. Lockhart (1992: 158-159), la palabra «huehueh» en los términos «huehuehtlalli» («tierra antigua»), «huehuehcalli» («casa antigua»), «huehuehmilli» («milpa antigua»), etc., debería entenderse como «patrimonial» $\mathrm{o}$ «heredado», con lo cual este tipo de posesión pertenecía a particulares o bien a corporaciones como, por ejemplo, el calpolli. Así, en realidad huehuehtlalli no debería permanecer en las manos de la familia u otra entidad desde tiempos inmemoriales y hay pruebas de que la tierra de este estatus era distribuida por los calpolli a sus miembros. Sin embargo, en los documentos se suele subrayar la continuidad asociada con la huehuehtlalli, sea su antigüedad («las milpas de nuestros padres y abuelos [antepasados], nuestros huehuehmilli»), o su futura posesión «por la eternidad» («su huehuehtlalli que va a dejar a sus parientes para que siempre coman y beban de ello»).

Los escritores nahuas se refieren frecuentemente tanto al pasado como al futuro con el uso de términos de parentesco. Los antepasados y descendientes se describen con unas metáforas basadas en el formato de multifrasismo, cuya variante más común, difrasismo, se compone de dos palabras, las cuales son dos elementos constituyentes del concepto que describen o bien, asociándose uno con otro, crean un nuevo significado. Así, a los huehuetqueh, o antepasados, se les llama «-nanhuan -tahhuan» («madres y padres de alguno»), «-cihhuan -colhuan» («abuelas y abuelos de alguno»), «-tahhuan -colhuan» («padres y abuelos de alguno»), «-achtonhuan -cohcolhuan» («bisabuelos y abuelos de alguno») (p.ej. Chimalpahin 2006: 111; Karttunen y Lockhart 1987: 132; Lockhart 1991: 95; Sahagún 1997: 237). Aunque los difrasismos como «padres abuelos» mencionan únicamente a dos generaciones, su sentido es más general y puede extenderse a tiempos muy remotos. Por ejemplo, en el siglo XVII un testador dijo:

\footnotetext{
3 «auh macihui yn amo huel momati yn ac yehuantin. yn techihuanime yn tetahuan catlehuatl yntoca yn otechiuhque yece iuh mitohua motenehua ca huel ixhuiuhtzitzinhuã yn tlacatl acamapichtli huell itech tlanticate ynic mitohua huehue pipiltin».
} 
«[esta tierra] hace tiempo la dejaron al morir mis padres, mis abuelos [mis antepasados], porque ellos iniciaron, ellos fundaron este gran altepetl de Cempoallan ${ }^{4}$ (Rojas, Rea y Medina 2000: 89).

Las combinaciones de varios términos de parentesco para referirse a los antepasados parecen interminables y a veces forman secuencias bastante largas, como por ejemplo «sus madres, padres, abuelos, abuelas, bisabuelos» ${ }^{5}$ (Sahagún 2012 VI: 64) o «nuestras abuelas, nuestros abuelos, bisabuelos, tatarabuelos, bisabuelas, ancestros» ${ }^{6}$ (Chimalpahin y Tezozomoc 1997: 60-61). El mismo concepto se manifiesta en las genealogías pictóricas, donde se registran unas generaciones múltiples de los ancestros masculinos y femeninos que forman una red compleja de la cual un descendiente puede trazar, de manera bilateral, su identidad, estatus, derechos y privilegios. Así, códices como la Genealogía de Zolin, la Genealogía de Tlatzcantzin o la Genealogía de los descendientes de Nezahuacoyotl demuestran estas líneas extensas o redes de conexiones de parentesco que dan profundidad y fuerza procedentes de las generaciones pasadas que refuerzan la posición y derechos de un descendiente concreto en el presente.

\section{Hacia una perspectiva intelectual: reevaluación de la tradición indígena y diálogo con Europa}

En el año 1564 fray Bernardino de Sahagún encontró apuntes en náhuatl tomados después de un debate de los frailes españoles con los nobles nahuas en 1524. Basándose en este texto, Sahagún y su equipo de ayudantes indígenas escribieron lo que hoy día conocemos como Colloquios y doctrina cristiana, considerado un testimonio de uno de los primeros intentos de convertir a la elite nahua al cristianismo (León-Portilla 1986: 19-20). La estructura de los «Colloquios» y el lenguaje sofisticado que utilizan tanto los frailes como los «in teteuctin in tlahtoqueh» («señores gobernantes»), evoca una forma tradicional de reunión entre dos grupos de huehuetqueh con el fin de presentar conjuntamente los huehuehtlahtolli. En esta convención se inscriben también los discursos del libro VI del Códice Florentino, recopilados en los años cuarenta del siglo XVI, que registran los huehuehtlahtolli como diálogos entre representantes de los huehuetqueh (p.ej. Sahagún 2012 VI: 189-196). El vocabulario empleado en estos textos enfatiza el intercambio no sólo de las palabras, sino también del «aliento» que contenía esencias divinas. Al responder, una persona devolvía el aliento y la palabra (es decir, discurso) a su interlocutor: «Contesta el interlocutor, devuelve la palabra, dice:» ${ }^{7}$ (Sahagún 2012 VI: 136), «iquién va a devolver, quién va a retomar vuestro aliento?» ${ }^{8}$ (Sahagún 2012 VI: 138). Según los colaboradores de Sahagún la misma forma adquirió la conversación del año 1524 :

\footnotetext{
4 «yquin canin oquimocahuylitiaque notahuan nocolhua canel yehuan oquitzintique oquimanque ynin huey altepetl Cenpualan».

5 «in jnaoan, yn jtaoan, in jculhoan in jcioan in jachtoa».

6 «tocihuan tocolhuan yn tachtõhuan yn tomintonhuan yn topiptonhuã yn tochichicahuan».

7 «Tlananqujlia in nonotzalo, qujcuepa in tlatolli: qujtoa».

8 «aqujn qujcuepaz, aqujn qujlochtiz amjhijotzin».
} 
«Luego uno de los señores gobernantes se puso de pie, saludó a los frailes. Devolvió su aliento, su palabra [su discurso] con pocas [palabras], una, dos, palabras. Dijo» ${ }^{9}$ (Sahagún 1986: 136).

Es imposible establecer hasta qué punto la forma que el equipo de Sahagún dio a los «Colloquios» en 1564 reflejaba la manera en la que los participantes indígenas percibían este intercambio de ideas en 1524. Seguramente la interpretación de estos textos como unos huehuehtlahtolli dio un valor adicional a la defensa de la tradición prehispánica, que los nobles presentaron en confrontación con el requisito de convertirse en cristianos. Guardando la humildad frente al nuevo Dios y sus portavoces españoles, los nahuas, sin embargo, se identifican como herederos de todos los tlahtohqueh mexica, subrayando la antigüedad e, implícitamente, el prestigio de su tradición:

«Aunque nosotros actuamos como señores, somos las madres y padres de la gente, ¿luego aquí, en vuestra presencia, hemos de destruir el antiguo costumbre del pueblo, que nuestros abuelos y abuelas [antepasados, los huehuetqueh del pasado] tenían en mucho, en favor del cual hablaban mucho y le daban honra los señores y gobernantes?» ${ }^{10}$ (Sahagún 1986: 138).

Lo mismo repiten los sacerdotes nahuas, refiriéndose a la historia de los mexica:

«¿Hemos de dañar nosotros el antiguo costumbre del pueblo? ¿El costumbre chichimeca? ¿El costumbre tolteca? ¿El costumbre colhuaca? ¿El costumbre tepaneca?» ${ }^{11}$ (Sahagún 1986: 152).

De esta forma, los sacerdotes reconocen la herencia cultural de los mexica, mencionando los grupos más importantes para la identidad del altepetl de Mexico-Tenochtitlan. Las referencias al pasado mito-histórico del altepetl sirven para demostrar tanto la fuente prestigiosa como la riqueza de la tradición indígena y así resistir a la perspectiva europea de la dominación cultural del cristianismo.

El proyecto de apreciación de la tradición nahua continuaba en las obras de los cronistas indígenas y mestizos que escribían a finales del siglo XVI y principios del siglo XVII. Don Hernando de Alvarado Tezozomoc y don Domingo de San Antón Muñon Chimalpahin Quauhtlehuanitzin, eran hombres educados según los estándares europeos y en su trabajo se servían tanto del idioma náhuatl como del español. Aquella competencia cultural doble se refleja en sus obras, las cuales, al intentar preservar y transmitir la herencia indígena, buscan un espacio para ella en el discurso historiográfico europeo. Tanto Chimalpahin (2003 II: 306) como Tezozomoc, en varios contextos se refieren a sus escritos con el término de huehuehamoxtli («libros antiguos» o en este caso mejor, tal vez, «libros de la tradición»). Tezozomoc no hace dudar de que su propósito es la continuación de la transmisión tradicional, aunque ya con los medios españoles (escritura alfabética):

\footnotetext{
9 «njman ce iehoâ in tetecutin tlatoque omoquetz, quîmotlapalhuj in teupixquq^ auh achitzin centêtlj ontêtlj ic oqujcuep in jmjhiiotzin in jntlatoltzin. quito».

10 «maciujn tehoâtin titecuchivâ titenâti, titetati, cujx nimâ tehoan njcan amjxpâtzinco tocôpolozq` jn veve tlamanjtiliztlj in qujveymattiuj in tocolhuâ in toçihuâ, in vel ipan tlatottiuj, in qujmaujcotitiuj, in tetecuti, in tlatoque».

11 «Auch [sic] cujx ie teoantin, toconitlacozq ${ }^{\wedge}$ in veve tlamanjtiliztli? in chichimeca tlamanjtiliztlj? in tolteca tlamanjtiliztli? in colhuaca tlamanjtiliztli? in tepaneca tlamanjtiliztli?».
} 
«Este antiguo discurso para arreglar la vida, este antiguo libro para arreglar la vida en México, se ha dejado a nosotros. Estas palabras son en verdad lo que guardamos para que otra vez también nuestros hijos, nuestros nietos [nuestros descendientes], nuestra sangre, nuestro color [nuestros descendientes nobles] que van a salir de nosotros, para que ellos también siempre lo guarden. Vamos a dejarlo a ellos cuando moriremos»» ${ }^{12}$ (Chimalpahin y Tezozomoc 1997: 61-63).

Pero, unas pocas líneas más abajo, afirma que quienes le proporcionaron este relato ya fueron cristianos: «aquí se terminan las palabras de los huehuetqueh, que fueron los primeros cristianos, que fueron los primeros estudiantes nobles» ${ }^{13}$ (Chimalpahin y Tezozomoc 1997: 63). Así, el estatus de los huehuetqueh como fuente y guardianes de la tradición, se le asigna a la primera generación después de la conquista: los nobles educados por los frailes españoles. Aunque la transmisión está realizada a través de la escritura, se sirve del mecanismo propio de la oralidad: la actualización o reinvención del contenido. Al mismo tiempo, la heterogeneidad de la «tradición» no impide su papel fundamental para conservar la identidad nahua.

El concepto de los huehuetqueh, como progenitores, fundadores y legitimadores, se conserva hasta la época colonial tardía. Un ejemplo de la continuación de esta tradición nos lo proporciona el titulo primordial de San Antonio Zoyatzinco (región de Amecameca, referido en el documento como Cihuatzinco Zoyatzinco), del siglo XVIII (AGN, Tierras 1665, exp. 5, ff. 166-182). Este manuscrito pertenece al género de textos fabricados por las comunidades indígenas con el propósito de proporcionar una fuente de legitimación de la posesión de tierras, del estatus socio-político de la comunidad y de su identidad, entendida en los términos más generales ${ }^{14}$. La narración se remonta a acontecimientos del siglo XVI situando la fundación y organización del pueblo entre los años 1555 y 1559 cuando se hizo la congregación, la división en barrios y la delimitación de límites:

«Cuando hubo sometimiento de la gente [la conquista], se hizo la congregación. Se hicieron cuatro barrios, con lo cual hubo desacuerdos. Todavía no había la peste o la tos y otras cosas más, la lepra. En ese momento, por todas partes cada uno de los altepetl ganó sus tierras, y se encargaron los nobles [del gobierno]» ${ }^{15}$.

La parte inherente del relato es su componente iconográfico que complementa y enriquece el texto alfabético, entrelazando y entretejiendo los elementos pictóricos y escritos. Este modo de expresión continúa una convención nahua prehispánica, donde los elementos iconográficos y glíficos están en un constante diálogo y se complementan mutuamente. El espacio principal lo ocupan las representaciones de dos per-

\footnotetext{
12 «auh ynin huehuenenonotzaliztlahtolli ynin huehuenenonotzalizamoxtlacuilolli mexico yn oticahuililotiaque yn huel topial ynin tlahtolli ynic no tehuantin oc ceppa yn topilhuan yn toxhuihuan yn teçohuan yn totlapallohuā yn totechcopa quiçazque ynic mochipa no yehuātin quipiezque. tiquincahuilitiazque yn iquac titomiquilizque».

13 «nican tlami yn intlahtol huehuetque yn achto christianosme catca yn achto momachtianime pipiltin catca».

14 Sobre el género de los títulos primordiales, véase Haskett 2010.

15 «yn iquac hualten ${ }^{\text {pa }}$ choloc mochiuh congrecansio nahui tlaxilacali mochiuh ynic omonanamitz ayemo o ca cocoliztli noson tlatlacciztli ca ocse tlamatli y teococoliztli yn ipan omomaceuh tlali y nohuiya cecen nalltepetl y momama tepilhuane».
} 


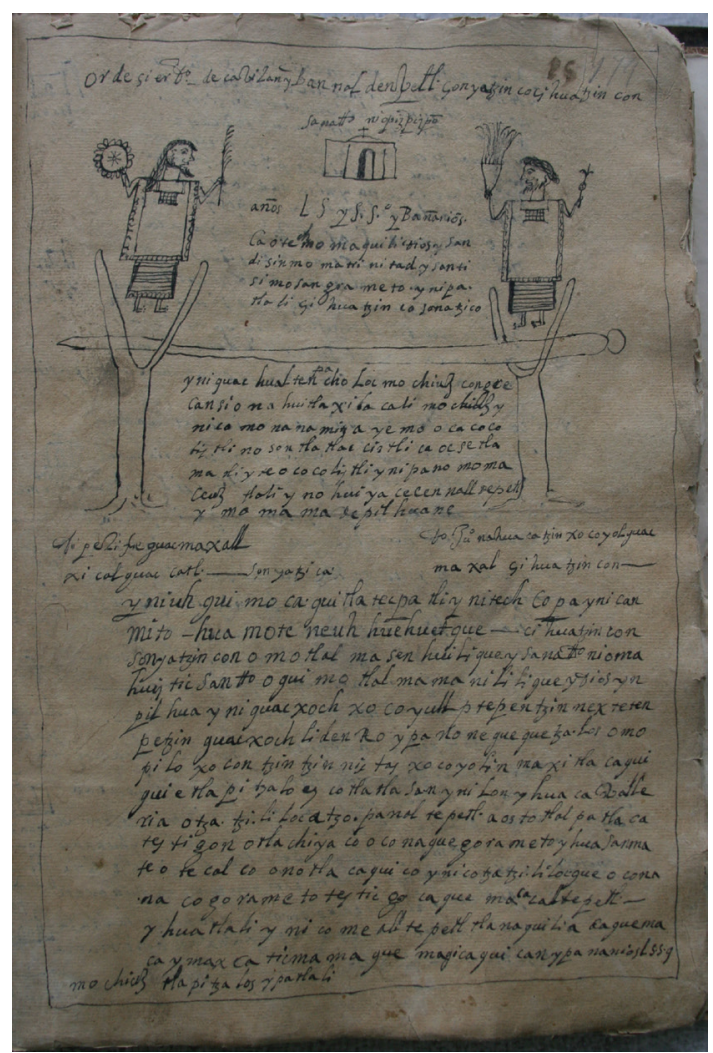

Figura 1: Escena de fundación de Cihuatzinco Zoyatzinco, Titulo primordial de San Antonio Zoyatzinco, Archivo General de la Nación, fondo Tierras 1665, exp. 5, fol. 179r.

sonajes fundadores con traje femenino (faldas y huipilli decorados) pero con barbas y peinado masculino y varios objetos en las manos (Figura 1): un escudo, una planta (o quizás originalmente una macana), un manojo de plumas o ramas y un posible cetro. La confusión de convenciones iconográficas, como el atavío femenino y los rasgos masculinos de los personajes, es bastante común en los documentos pictóricos del periodo colonial tardío y sobre todo los del género de los títulos primordiales. Por otro lado, tampoco es imposible que los rasgos femeninos de los fundadores masculinos aludan al nombre del pueblo, Cihuatzinco, «El lugar de mujeres venerables». Cada uno de los fundadores aparece directamente encima de un árbol bifurcado. Los dos arboles están conectados por una vara puesta horizontalmente sobre las bifurcaciones, sugiriendo una cerca, quizás demarcación de una frontera, división o límite del terreno. El texto escrito debajo identifica a los protagonistas como $D^{o}$ pelife quacmaxall xicalquaccatl sonyatzica y $D^{o} J u^{o}$ nahuacatzin xocoyol quacmaxal çihuatzincon. La palabra repetida en los dos nombres cuauhmaxalli, horqueta, sin duda se refiere a la imagen pictórica, pero posiblemente también a una «bifurcación» del altepetl, la división interna en las partes de Zoyatzinco y Cihuatzinco. De acuerdo con esta interpretación, probablemente haya que leer en orden un poco diferente el nombre del primero de los fundadores (está dividido en dos renglones, pero el orden puede ser vertical): $D^{o}$ pelife xicalquaccatl quacmaxall sonyatzica, Don Felipe Xicalquaccatl, 
Figura 2: Los fundadores de Cihuatzinco Zoyatzinco, Titulo primordial de San Antonio Zoyatzinco, Archivo General de la Nación, fondo Tierras 1665 , exp. 5, fol. $178 \mathrm{v}$.

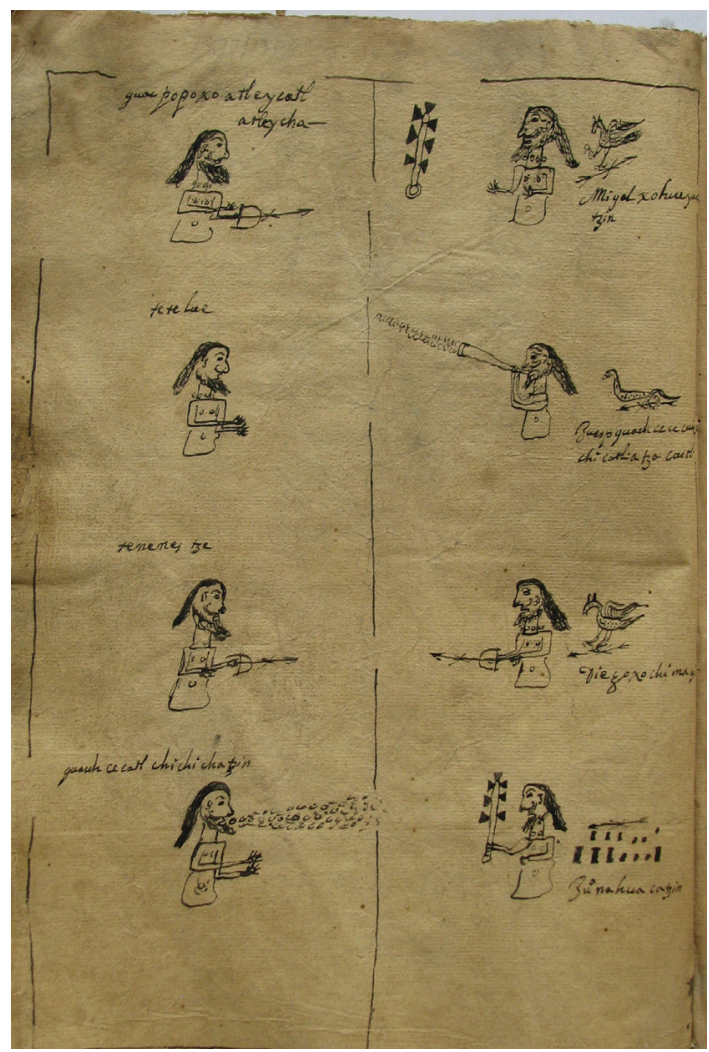

Quauhmaxalli de Zoyatzinco, cuyo contraparte en la otra división sería Don Juan Nahuacatzin Xocoyol, Quauhmaxalli de Cihuatzinco.

El texto a continuación hace una referencia directa a los huehuetqueh de Cihuatzinco Zoyatzinco quienes «ganaron las tierras» del pueblo. Aludiendo a la conquista de las tierras, se describe la delimitación y establecimiento de las fronteras así como una ceremonia de toma de posesión acompañada por la música, el disparo de armas y la presentación de caballería, todo ello reforzado por la asistencia de los representantes de algunos de los pueblos vecinos. Como en los siglos anteriores, son los «ancianos» los que constituyen una fuente de legitimidad histórica y orden para sus descendientes y su altepetl:

«De esta manera se entiende lo que se ha puesto en órden en cuanto a lo que se dice aquí. Se ha mencionado que los ancianos de Cihuatzinco Zoyatzinco ganaron las tierras de San Antonio, el santo ilustre. Los hijos de Dios extendieron las tierras hasta el lindero de Xocoyoltepetzin y Nextetepetzin. En el lindero fue levantado y se colgaron frutas y acedera blanca. Vengan a escuchar. Ya se tocan los instrumentos, se disparan las escopetas, hay caballería. Se pregonó en el altepetl de Atzompan. La gente de Aostotlalpan vinieron a hacer presencia como testigos y tomaron juramento. Y los de San Mateo Tecalco también vinieron a escuchar, acudiendo al pregón, y tomaron juramento como testigos. 'Sí, es así: el altepetl y la tierra'. El segundo altepetl responde, 'Sí, es así, es su propiedad. Nosotros nos encargamos. Que lo escuchen’. Se hizo en el año 
1559. Se tocarán los instrumentos sobre el terreno» ${ }^{16}$ (AGN, Tierras 1665, exp. 5, fol. 179r).

En el folio anterior del documento (Figura 2) se representa a los participantes en la toma de posesión tocando instrumentos, cantando o pregonando y portando armas prehispánicas como el macuahuitl o «macana» y el arco con flechas, quizás aludiendo a la ceremonia antigua de flechamiento que acompañaba los actos de fundación y toma de posesión de tierras.

\section{Diálogo con Europa}

La familiaridad relativa con la antigüedad clásica entre la elite indígena, y especialmente su conciencia de que los mismos habitantes de España fueron conquistados y convertidos al paganismo por los romanos, abría las puertas a unas interpretaciones no siempre favorables para los españoles (Gruzinski 2002: 95). En particular, en la percepción de los intelectuales nahuas, el paralelismo con la antigüedad europea sin duda prestaba más valor y prestigio a la tradición pagana indígena. Estas ideas reverberan en los escritos de Chimalpahin, uno de los más destacados intelectuales nahuas de finales del siglo XVI y principios del XVII. Un punto de partida para este tipo de discurso parece ser la identificación de los ilustres griegos y romanos, el mismo Platón, como paganos: «También los dichos gentiles, aunque idolatras, nos dan mandamientos y ejemplos relacionados con esto, como Platón» ${ }^{17}$ (Chimalpahin 2003 I: 30). Del mismo modo, afirma el autor indígena, Ovidio «era pagano [tlateotocani] y no conocía al Dios único» (Chimalpahin 2003 II: 18). Al usar el mismo término en referencia tanto a los grandes de la antigüedad europea, como a sus propios antepasados, conscientemente o no, Chimalpahin crea un paralelo indiscutible entre los dos mundos, que se convierte también en una fuente de (re)valoración del pasado indígena. El lazo construido por el cronista va incluso más allá al identificar a los gigantes descritos por Ovidio con los quinametin, los seres gigantescos de la tradición mítica nativa, habitantes del mundo o época anterior (Chimalpahin 2003 II: 18). Es también notorio que, aunque el autor nahua emplea los mismos vocablos, gentiles e idólatras, para referirse a sus propios antepasados, ese uso es escaso en comparación con la denominación huehuetqueh o huehuetqueh chichimeca, mucho más frecuente. Éstos últimos, como los griegos y romanos, también salieron de la primera generación humana del Viejo Mundo (Chimalpahin 2003 I: 28):

\footnotetext{
16 «yn iuhqui mocaqui tlatecpatli yn itechcopa y nican mitohua moteneuh huehuetque - cihuatzincon soyatzincon omotlalmasenhuilique y san att ${ }^{\circ}$ nio mahuiztic santto oquimotlalmamanililique y Dios ynpilhua yn iquacxoch xocoyullptepentzin [xocoyoltepetzin] nextetenpetzin quacxoch lidenro ypan onequequetzalos omopilo xocontzintzin nistasxocoyolin [iztac xocoyolli] ma xitlacaquiqui e tlapitzalo ezcotlatlasanynilon yhua caovalleria otza.tzi.liloc atzo.pan altepetl aostotlalpatlaca testigon otlachiyaco oconaque gorameto yhua san mateo tecalco o no tlacaquico ynic otzatzililocque oconananaco gorameto testicgo ca quema ${ }^{\text {ca }} \mathrm{c}$ altepetl - yhua tlali ynic ome altepetl tlanaquilia ca quema ca ymaxca ticmamaque ma gicaquican ypan anios 15.5.9 mochiuh tlapitzalos ypa tlali».

17 «Auh ca no yehuantin yntla yn tlateotocanime yn mitohua motenehua gentiles ompa no techmaca tetlaquauhnahuatilli yn machiyotl octacatl yn itechpa yuhqui in; yn iuhqui yehuatl ytoca Platón».
} 
«salimos y nacimos de la primera generación humana, de ella descendemos todos que somos los hombres sobre la tierra en el mundo, aunque fueron paganos e idolatras los de quienes descendemos, quienes nos engendraron a nosotros los habitantes de México Tenochtitlan ${ }^{18}$.

Así, las raíces de los habitantes de América nacen de la tradición bíblica (Chimalpahin 2003 I: 38): de «nuestro primer padre Adán y nuestra primera madre Eva» (achto totatzin Adán yuan yn achto tonantzin Eva), «nuestros primeros padres Adán y Eva» (yn achto totatzitzinhuan Adam yhuan Eva). Esta visión de la historia compartida, se refuerza por los intentos numerosos de Chimalpahin para correlacionar la historia bíblica y cristiana con la cuenta y tradición histórica indígenas. Por otro lado, el cronista también está, de cierta manera, negociando el lugar del Nuevo Mundo en el orden global de la tierra y en referencia a los demás continentes del Viejo Mundo. Así, siguiendo el relato de Enrique Martínez, Reportorio (véase Schroeder 2010: 115), admite que de las cuatro partes del mundo Europa es la más importante: Auh yn occenca hueycan yn ipan in nauhtetl tlalli ca ompa yn ipan Europa («La principal de estas cuatro tierras es Europa»; Chimalpahin 2003 I: 64-65). Además, afirma también que aunque los antiguos nunca supieron con certeza de la existencia del Nuevo Mundo, este sobrepasa a todos los demás continentes: Auh occenca quinpanahuia occenca tlamiahuayotia yn nican ypan cemanahuatl yn tlalli yn çaço cacatlehuantin ceme yn occequi yexcanme tlalli ( $\mathrm{Y}$ Y esta tierra del mundo sobrepasa y excede cualquiera de las demás tres tierras»; Chimalpahin 2003 I: 68-70).

Refiriéndose nuevamente a sus antepasados, traza la descendencia de los antiguos chichimecas de uno de los hijos de Noé, aunque no tiene claro de cuál de ellos exactamente provienen los huehuetqueh chichimeca, o sea los antiguos chichimecas, no obstante indica que «se cree que todos somos los hijos del primero padre Adán y de Noé porque de ellos descienden los antiguos chichimecas quienes mencionamos y quienes desembarcaron en Aztlan» (Chimalpahin 2003 I: 314) ${ }^{19}$. La correlación con la historia cristiana está omnipresente: sitúa el establecimiento de sus antepasados 18 años después de la ascensión al cielo de Jesucristo (Chimalpahin 2003 I: 312), pero al mismo tiempo rechaza la hipótesis de que los chichimecas descienden de los judíos dispersos después de la destrucción de Jerusalén, dado que según sus cálculos se asentaron algún tiempo antes de este acontecimiento. Sin embargo, la analogía y un lazo con la historia bíblica se hacen mucho más profundos cuando el cronista nahua enuncia que los huehuetqueh en Aztlan hablaban un sólo idioma y todavía no ha cambiado su habla (çan oc centetl yn itlahtol catca, ayemo mocuecuepa yn tlahtolli; Chimalpahin 2003 II: 314), mientras que la separación étnica, acompañada por la división de lenguas, ocurrió después de abandonar el lugar de origen. Esta analogía directa con la Torre de Babel se hace hasta más explícita en la Séptima Relación, donde Chimalpahin identifica el lugar mítico de origen con Tlapallan Nonohualco, refiriéndose a este sitio como tlahtolli ymocuehcuepyan; «lugar donde se revolvieron las

\footnotetext{
18 achto tlacamecayotl yn itech otiquizque otitlacatque ytech otitlacamecayohuaque yn tixquichtin yn ipa ceman»ahuatl titlaca tlalticpac, maço nellihui yn gentiles yn [tlateoto]canime yn intech tiquiça yn otech[tla] catillico yn tehuantin y nican titlaca Mexico Teno[chti]tlan...».

19 «Auh yece yuhca tlaneltoquiliztli ca moch tipilhuan yn achto totahtzin Adán yhuan yn Noé, ca oncan yntechcopa hualquiça yn otiquinteneuhque yn huehuetque chichimeca yn oncan atenquiçaco Aztlan».
} 
lenguas». Los ancianos tlacochcalcas, sus antepasados, dejaron allí su primera lengua y adoptaron una nueva. Sin embargo, al descubrir la discrepancia entre la cronología indígena de los tlacochcalcas y la bíblica, Chimalpahin concluye que sólo hubo una confusión de lenguas en la historia del mundo que ocurrió cuando los habitantes de Babilonia construían su torre. Aunque la cuenta antigua indiana «no concuerda con la cuenta cristiana porque omite muchos siglos» (yn inhuehuexiuhtlapohualiz tlacochcalca amo quinamiqui yn chr[ist]iano xiuhtlapohualli ca cenca miec tzonxippa tlacahua), el intelectual indígena da más credibilidad a la tradición bíblica que, a su vez, explica mejor y proporciona el contexto correcto para este elemento de la tradición histórica indígena (Chimalpahin 2003 II: 314).

\section{Una perspectiva pragmática}

Al lado de los proyectos culturales existía otro campo donde los conceptos de la tradición, antigüedad y prestigio fueron extensamente explorados y empleados por los nahuas: las negociaciones de diferente índole con el sistema sociopolítico de la Nueva España. Estas podían tomar las formas de una lucha por tierras y privilegios, reducción de tributo, reconocimiento de méritos así como confirmación de estatus, convirtiéndose en una serie de estrategias y procedimientos desarrollados a un nivel de un individuo/familia o de una comunidad entera. Así, una gran parte del «teatro» político local se llenó con las aspiraciones de conseguir la posición de una cabecera o ser elevado a un estatus de gobernante con derecho de montar a caballo y llevar armas y confirmar la posesión o adquisición de una propiedad privada. Una preocupación frecuente era también legitimar las delimitaciones de fronteras del pueblo o lograr reconocimiento, tanto de los méritos personales como las virtudes de un altepetl, frecuentemente basados en las supuestas actuaciones en la conquista como aliados de los españoles o en un papel desempeñado en la cristianización.

La dimensión pragmática y práctica era un componente fundamental de todos estos esfuerzos. Empezando ya en las primeras décadas después de la Conquista, los señores indígenas escribían cartas al rey de España, esperando mejorar la situación económica de sus altepetl. Entre los argumentos presentados en estas peticiones destacan dos estrategias: enfatizar el estatus importante del altepetl antes de la llegada de los europeos y subrayar el apoyo que Cortés recibió de manos del pueblo durante su guerra contra México Tenochtitlan. Así, el tlahtoani de Tlacopan, don Antonio Cortés, escribe en 1552:

«quando el marques del valle vino a esta tierra luego de grado y voluntad Recebimos la palabra y fe de n[uest]ro señor Jesu Cristo y ansi mesmo Recebimos a .v. magt. por $\mathrm{n}$ [uest]ro Rey y señor y fuimos en ayudar a los españoles quando çercaron y conquistaron a mexico ansi mesmo hago saber a .v.c.c.m. como mi padre que era señor quando vino el marques del valle y mis aguelos señores de tlacopan etc. nunca tributaron a mexico ni a otro señor ninguno con vn tomin ni vn grano de cacao» (AGI, México 96).

A pesar del formato europeo de la petición, en el uso de la palabra «abuelos» para referirse a los antepasados muy remotos se refleja la manera indígena de hablar sobre 
el pasado. El difrasismo completo se encuentra en otro documento, firmado por don Francisco Verdugo, el tlahtoani de Teotihuacan:

«el y sus padres. y abuelos. deçienden. del señor. y señores. que son. y fueron de la çiudad. de tezcuco. cabeça. de la gran. trra. de la nueua España» (AGI, México 96).

En este caso los autores de la petición no sólo presentan al tlahtoani como el último enlace en la cadena de señores ilustres, sino que también se aprovechan de la ignorancia supuesta de los españoles, sugiriendo que Texcoco gozaba de un estatus más grande que Tenochtitlan. En lo que sigue, mencionan al tlahtoani texcocano Nezahualcoyotl, el cual, según ellos, pertenecía a la dinastía que gobernaba las tierras de la Nueva España antes de la venida de los mexica.

La preocupación de los «caciques» por la economía y prestigio de los altepetl iba de la mano con la lucha por su estatus individual en la sociedad colonial. Tanto don Antonio Cortés, como don Francisco Verdugo solicitaban al rey los escudos de armas que les permitirían confirmar su posición dentro de la elite. Los diseños reflejaban la compleja identidad de la nobleza indígena, combinando los símbolos cristianos con la iconografía del poder española y con las representaciones de cosmovisión y tradición política prehispánicas ${ }^{20}$. Al mismo tiempo, las elites intentaron conservar su estatus privilegiado como propietarios de la gran cantidad de tierras cultivables. Las peticiones al rey forman nada más una pequeña parte del corpus de documentos que registran la lucha de los nobles indígenas no sólo con europeos, sino también con sus vecinos, vasallos e incluso parientes. El pleito tlatelolcano del año 1558, citado anteriormente, es un ejemplo excelente de estrategias utilizadas frente a las autoridades coloniales para autentificar los derechos a la propiedad. Las partes del pleito son Pablo Huitznahuatl, el cual se presenta como descendiente de los tlahtohcapipiltin (miembros de la dinastía real) de Tlatelolco y Bernardino Cozcaquauh, junto con su hermana, aparentemente de una descendencia más humilde. Pablo se apoya en una narrativa típica para las elites indígenas del siglo XVI con la que lograban ganar en los pleitos una posición más fuerte, subrayando su pertenencia a los niveles más altos de la aristocracia (Kellog 1995: 26). Con este propósito, Pablo proporciona una enumeración detallada de sus antepasados:

«En verdad, ésta es mi tierra heredada. La vinieron a ganar mis abuelos [antepasados], Epcoatzin y Chicopachmani. Ellos, Epcoatzin y Chicopachmani murieron hace ochenta años. En estos tiempos ya andaban guardando la tierra. El nombre de mi abuelo fue Xocotlacuatzin, el cual murió hace sesenta años, en los tiempos de Ahuitzotzin. Éste [también] guardaba [la tierra]. Mi padre, el cual murió hace veinte años, la guardaba [también]. Ahora soy yo quien guarda la tierra todo el tiempo» $»^{21}$ (AGN Tierras vol. 17, parte $2^{\mathrm{a}}$, f. $13 \mathrm{r}$ ).

Cabe señalar que Epcoatzin fue un personaje muy importante en la jerarquía política del México prehispánico, porque era el abuelo de Cuauhtemoc, el tlahtoani de

\footnotetext{
20 Sobre los escudos de armas indígenas, véase Castañeda de la Paz 2006, 2008.

21 «ca vel novevetlal. quimacevaco y nocolvā yepcovatzīn chicopachmani ynīn ya napovalxitl ŷ mimique yepcovatzin chicopachmani auh ynin ya ypa quipixtivi y tlali auh y nococol ytoca Socotlaquatzin ŷ miqui ya nepovalxivitl ypā avitzotzin auh ynin oquipixta auh y nota ya cenpovalxivitl. yn omiqui oquipixta. auh yn axcā ya nevatl. mochipa ya nicpiya $\hat{y}$ tlali».
} 
Tenochtitlan (Chimalpahin y Tezozomoc 1997 I: 78). De esta manera, al igual que don Antonio Cortés y don Francisco Verdugo, Pablo Huitznahuatl se coloca como descendiente de un linaje prestigioso. Se sirve de sus antepasados no sólo para contar la historia de un pedazo de tierra guardado por su familia, sino también para aumentar su credibilidad frente a la Corte, subrayando la disparidad entre él mismo y sus adversarios que no gozaban de los ancestros tan ilustres.

Bernardino Cozcaquauh y su hermana no podían presentarse como descendientes de una dinastía real, ni demostrar un vínculo con la tierra que se remontaría a tiempos prehispánicos, ya que según su versión su familia había adquirido la milpa en el año 1521. Esta fecha nos acerca al origen supuesto del conflicto, que podía resultar de la apropiación de la propiedad de los nobles tlatelolcanos, vencidos y encarcelados por Cortés después de la caída de Tenochtitlan. Pero Bernardino tuvo una base fuerte para su argumentación: su posesión se originó en tiempos de la Conquista, es decir, en el punto de partida del sistema colonial. Desde este momento él también fue capaz de demostrar la continuidad de herencia que seguía la costumbre indígena: su hermana le dejó la tierra al morir para que la guardase hasta que su sobrino regresase a Tlatelolco. La versión de Bernardino está confirmada por los huehuetqueh de su pueblo, los cuales proporcionan información muy detallada sobre el precio de la tierra, los participantes y otras circunstancias de la transacción. Parece muy probable que para esta ocasión se servían de unas memorias pintadas de la comunidad. Pablo Huitznahuatl también presenta a los testigos que describen la cadena de herencia desde los tiempos de la Conquista, pero ninguno de ellos se identifica como un huehueh. Es posible que en opinión de Pablo la autoridad de su linaje prestigioso fuera suficiente para autentificar su versión frente a los jueces españoles. Sin embargo, la estrategia de Bernardino demuestra que las referencias al pasado prehispánico no fueron el único modo de utilizar la tradición. Para los nahuas del siglo XVI, la autoridad de los huehuetqueh y la memoria común de la que disponían, igual que los antepasados prestigiosos, eran bastante prometedores para conseguir el éxito en los pleitos.

En su testamento del año 1610 (Rojas, Rea y Medina 2000: 74-95), don Juan Bernardino, fiscal del pueblo de Cempoallan, se refiere a las tierras que deja a los descendientes como herencia recibida de los grandes antepasados: «un gran noble nuestro bisabuelo Ixtlilxochitl, merecedor (conquistador) de la tierra» (yn huey pilli ça notachcocoltzin Yxtlixuchitl tlalmaceuhhqui). De Ixtlilxochitl deriva «la tierra de patrimonio» o de cacicazgo identificada como tlahtohcatlalli, de acuerdo con el concepto prehispánico de la tierra asignada al tlahtoani, el gobernante de un altepetl. Mas adelante, el testador afirma que se la dejaron «mis abuelos, mis padres pues era una propiedad de un gran noble Ixtlilxochitl» (yn nocolhuan yn notahu[an] ca yaxcatzin catca yn huey pili Yxtlixuchitl), «los ancianos, los nobles, los conquistadores de tierra» (yn huehuetque yn pipilti yn tlalmaceuhque). Así, en la retórica de los documentos cotidianos alfabéticos que siguen un modelo español, como los testamentos, los antepasados ilustres se convierten en la fuente principal de la legitimación de la posesión de la tierra, pero la transferencia de autoridad sobre el terreno se refuerza también por el poder español que actúa en el nivel legal. Este parece complementar el ámbito de poder asociado con el nivel de tradición y herencia indígena: 
«les dejo todo que era la propiedad de mi padre, mi abuelo, mi bisabuelo Ixtlilxochitl el gran conquistador, que yo agarre lo que era su propiedad, tome todos los documentos de tierra, comisiones, las cuatro comisiones que le dio nuestro rey Carlos Quinto» ${ }^{22}$.

Hacia finales del periodo colonial, el papel de la legitimación por el poder español se desarrolla más en los Títulos Primordiales que hacen referencia a Hernán Cortes, Antonio de Mendoza, Luis de Velasco y otros personajes importantes de las primeras décadas después de la conquista. No obstante, este pasado reciente de la conquista fue empleado para lograr beneficios muy concretos y tangibles ya pocas décadas después de la llegada de los españoles. Llama la atención que en lugar de referirse a este contexto histórico como fuente de privilegios recibidos, los actores políticos indígenas acentúan su papel como agentes directos y activos que lograron y merecieron los beneficios concretos a través de su actitud bastante independiente y méritos específicos. Entre varios escritos de la nobleza indígena basados en esta argumentación, destaca la carta del cabildo de Huexotzinco al rey escrita en el año 1560 (Anderson, Berdan y Lockhart 1976: 176-191), pues en ella los huexotzincas se presentan como el primer pueblo que recibió a los españoles y se convirtió voluntariamente en súbdito de Cortés gracias a la inspiración y alumbramiento divino. Es más, utilizando la misma retórica, desacreditan a sus rivales políticos, los tlaxcaltecas, quienes supuestamente decidieron ayudar sólo porque ellos, los huexotzinca, insistieron y les lograron convencer. Aun así, los tlaxcaltecas hicieron guerra a los españoles durante 15 días, mientras que, al contrario, los huexotzinca ayudaron a los españoles en la conquista, participando tanto en el asedio de Tenochtitlan, como en el de otras regiones de Mesoamérica, siendo los únicos que acompañaron a los españoles en todas sus campañas militares y nunca les abandonaron. Igualmente, de su propia voluntad recibieron a los franciscanos y la fe cristiana, al contrario de los infames tlaxcaltecas quienes demostraron resistencia, con lo cual varias personas fueron colgadas o quemadas por practicar idolatría. Como se esperaría de los fieles cristianos, encomiendan su petición al fraile, un intermediario y aliado, lo que refuerza la posición de la nobleza local indígena como partidarios de la iglesia cristiana. Toda esta argumentación y autopresentación, basada en la visión subjetiva de la historia reciente, sirve para negociar una favorable reducción del tributo. El razonamiento se refuerza con un argumento que se remonta al pasado prehispánico: el supuesto hecho de que antes de la conquista la nobleza local no tributaba a nadie. Ahora, por no respetar una orden antigua por la administración española, los descendientes de nobles prehispánicos corren en el riesgo de desaparecer por las causas de explotación excesiva.

\section{Conclusiones}

En el presente trabajo hemos bosquejado la trayectoria del concepto nahua de tradición a través de varios tipos de discurso representados por varios géneros literarios.

\footnotetext{
22 «ca mochi niquincahuylitiuh yn itlatquitzin yn iaxcatzin ocatca yn notatzin nocoltzin nachcocol Yxtlixuchitl huey tlalmaceuhque mochi onictzitziqui ticatca yn yntlatquitzin yn iaxcatzin ocatca ca mochi onictzitziquitoca yn oquimomaquili yn totlatocatzin yn huey Carlos Quinto yn mochi tlalamatl yn comixiontzi nahuy comixiontzitzi».
} 
Este concepto parece surgir de la cosmovisión nahua y su aspecto ideológico más importante: la circulación de esencias vitales en el mundo y su acumulación en los seres vivos. Los huehuetqueh, «ancianos» o «antepasados», llenos de la esencia caliente, son al mismo tiempo fuentes y portadores de tradición, existiendo por un lado en el pasado mítico y por otro lado en el presente. Así, reflejan los rasgos característicos para la cultura nahua: tiempo cíclico y la dominación de oralidad sobre escritura permiten una constante readaptación de tradición sin perder la creencia en su antigüedad inmemorable. El concepto de los huehuetqueh es también, como hemos demostrado, una noción clave para ahondar en la comprensión del discurso multifacético desarrollado por los miembros de la nobleza indígena en la época colonial. Es el papel universal de los antepasados el que une todas las esferas y niveles del diálogo con el pasado indígena, historia europea y los desafíos sociales, políticos y culturales de la realidad colonial. La aplicabilidad de este concepto radica no sólo en las implicaciones pragmáticas y prácticas que surgen de la legitimación política y económica, sino también en su profundidad conceptual y su importancia para la pervivencia de las comunidades nahuas. Hacia finales del periodo colonial, los huehuetqueh siguen con su papel de núcleo de la identidad indígena, aunque se modifica el modo de desempeñarlo. En los siglos XVI y XVII florece la literatura nahua que abarca géneros hasta entonces únicamente orales, como los huehuehtlahtolli. Los «discursos de tradición» ya no se asocian sólo con la noción del precioso aliento, portador de esencias divinas, sino también con la escritura alfabética: los «libros de tradición». El acceso a las tradiciones mito-históricas del Viejo Mundo culmina en proyectar el concepto indígena al contenido europeo, retro-alimentándolo y revalorizando al primero de ellos con una fuente adicional de legitimación. El punto de origen se traslada desde las cuevas de Chicomoztoc al Edén o, según otras perspectivas, a la llegada de Cortés y el primer bautismo de los señores nahuas.

La llamada por nosotras «perspectiva intelectual», reflejada en los escritos de la elite, no existía, sin embargo, en el aislamiento del discurso más práctico que encontramos en los documentos cotidianos. Los problemas mundanos de nobleza indígena -la lucha por tierras y privilegios, la negociación del espacio ideológico con los españoles- formaban también parte de la vida de los intelectuales. Por otro lado, la continuidad y el flujo de ideas alimentaban estrategias muy pragmáticas asociadas con la defensa de los intereses de los nobles. Fue otra dimensión del diálogo que mantenía la forma dinámica del concepto de la tradición: entre lo ideológico y lo práctico. Este último aspecto revela, como el primero, la adaptación de nociones europeas y la continuación de conceptos indígenas en el constante proceso de buscar similitudes entre ambos sistemas y espacios para la expresión cultural. Pero a pesar de esta dinámica de modificaciones y traslados, el papel fundamental de tradición y sus portadores (sea los huehuetqueh, libros escritos o manuscritos pictóricos), permaneció igual: conservar el «antiguo» modo de vida para las generaciones que vienen. Conservar las tierras, creencias, el sentido de pertenencia, el estatus de la comunidad, la base fuerte que no va a desintegrarse bajo las circunstancias del periodo colonial. Conservar la memoria y la identidad.

AGRadecimientos: Esta investigación fue apoyada por un subsidio del Centro Nacional de Ciencia en Polonia, concedido en la decisión DEC-2011/01/B/HS3/02185. 


\section{Referencias documentales}

AGI (Archivo General de Indias, Sevilla)

Audiencia de México 96: «Carta de don Antonio Cortés Totoquihuaztli al emperador Carlos $\mathrm{V} \gg, 1552$.

México 96: «Carta de don Francisco Verdugo Quetzalmamalitzin al rey Felipe II», 1558.

AGN (Archivo General de la Nación, México)

Tierras, vol. 17, parte $2^{\mathrm{a}}$, exp. 4, f. 11r-14v: «Proceso de don Pablo Uitznauatl y Bernardino Cozcaquauh, indio maceualli», 1558.

Tierras, vol. 1665, exp. 5, f. 166r-182v: «Título primordial de San Antonio Zoyatzinco».

\section{Referencias bibliográficas}

Anderson, Arthur J. O., Frances Berdan y James LockHart

1976 Beyond the Codices. The Nahua View of Colonial Mexico. Berkeley, Los Angeles, Londres: University of California Press.

Castañeda de la Paz, María

2006 «Escudos de armas tlaxcaltecas: iconografía prehispánica y europea». Arqueología mexicana 14(82): 68-73.

2008 «Apropiación de elementos y símbolos de legitimidad entre la nobleza indígena: el caso del cacicazgo tlatelolca». Anuario de estudios americanos 65(1): 21-47.

Chimalpahin, Domingo Francisco de San Antón Muñón Cuauhtlehuanitzin

2003 Las ocho relaciones y el Memorial de Colhuacan, Rafael Tena, trans. y ed. México: Conaculta.

2006 Annals of His Time, James Lockhart, Susan Schroeder y Doris Namala, trad. y eds. Stanford: Stanford University Press.

Chimalpahin, Domingo Francisco de San Antón Muñón Cuauhtlehuanitzin y Hernando de Alvarado Tezozomoc

1977 «Mexican History or Chronicle», en Codex Chimalpahin, Susan Schroeder y Arthur J.O. Anderson, trad. y eds., vol. 1, pp. 27-178. Norman: University of Oklahoma Press.

GeERTz, Clifford

1999 Conocimiento local. Ensayos sobre la interpretación de las culturas. Barcelona: Paidós.

GrUZINSKI, Serge

2002 The Mestizo Mind. The Intellectual Dynamics of Colonization and Globalization. Nueva York: Routledge Taylor \& Francis Group.

HASKETT, Robert

2010 «Primordial titles» en Sources and Methods for the Study of Postconquest Mesoamerican Ethnohistory. Documento electrónico, http://whp.uoregon.edu/Lockhart/, con acceso el 1/7/2014.

KARTTUNEN, Frances y James LOCKHART

1987 The Art of Nahuatl Speech. Los Angeles: UCLA Latin American Center Publications. 
KellogG, Susan

1995 Law and the Transformation of Aztec Culture, 1500-1700. Norman: University of Oklahoma Press.

LEÓN-Portilla, Miguel

1986 «Estudio introductorio» en Coloquios y doctrina cristiana, Miguel León-Portilla trad. y ed., pp. 15-36. México: Universidad Nacional Autónoma de México.

LOCKHART, James

1991 Nahuas and Spaniards. Los Angeles: Stanford University Press.

1992 The Nahuas after the Conquest. Stanford: Stanford University Press.

1999 Of Things of the Indies: Essays Old and New in Early Latin American History. Stanford: Stanford University Press.

López Austin, Alfredo

1984 Cuerpo humano e ideología, 2 vols. México: Universidad Nacional Autónoma de México.

Molina, fray Alonso de

1977 Vocabulario en lengua castellana y mexicana y mexicana y castellana, 2 vols. México: Editorial Porrúa.

RoJAs, José Luis de

2010 Cambiar para que yo no cambie. La nobleza indígena en la Nueva España. Buenos Aires: Editorial SB.

Rojas Rabiela, Teresa, Elsa L. Rea López y Constantino Medina Lima

2000 Vidas y bienes olvidados, vol. 3. México: Consejo Nacional de Ciencia y Tecnología, Historias Ciesas.

Ruíz DE Alarcón, Hernando

1984 Treatise on the Heathen Superstitions that Today Live among the Indians Native to this New Spain, 1629, James Richard Andrews y Ross Hassig, trad. y eds. Norman: University of Oklahoma Press.

SAHAGÚN, fray Bernardino de

1986 Coloquios y doctrina cristiana, Miguel León-Portilla trad. y ed. México: Universidad Nacional Autónoma de México.

1997 Primeros Memoriales, Thelma D. Sullivan y Henry B. Nicholson, trad. y eds. Norman: University of Oklahoma Press.

2012 The Florentine Codex, 12 vols, Charles E. Dibble y Arthur J.O. Anderson, trad. y eds. Santa Fe: The School of American Research.

Schroeder, Susan, ed.

2010 The Conquest All Over Again. Portland: Sussex Academic Press.

SETH, Vanita

2010 Europe's Indians: Producing Racial Difference, 1500-1900. Durham: Duke University Press. 